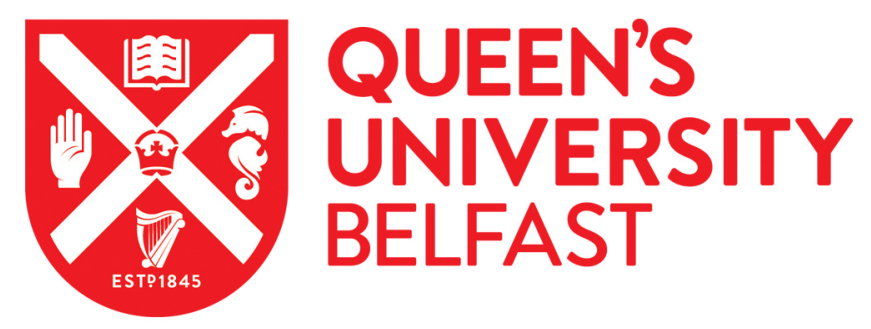

\title{
Perspectives of undergraduate module convenors at a South African academic institution on medical student training in evidence-based health care: a qualitative study.
}

Young, T., Rohwer, A., Volmink, J., \& Clarke, M. (2015). Perspectives of undergraduate module convenors at a South African academic institution on medical student training in evidence-based health care: a qualitative study. South African Family Practice, 57(6), 353-359. https://doi.org/10.1080/20786190.2015.1090689

Published in:

South African Family Practice

Document Version:

Publisher's PDF, also known as Version of record

Queen's University Belfast - Research Portal:

Link to publication record in Queen's University Belfast Research Portal

\author{
Publisher rights \\ (C) 2015 The Authors. \\ This manuscript is distributed under a Creative Commons Attribution-NonCommercial-NoDerivs License \\ (https://creativecommons.org/licenses/by-nc-nd/4.0/), which permits distribution and reproduction for non-commercial purposes, provided the \\ author and source are cited.
}

\section{General rights}

Copyright for the publications made accessible via the Queen's University Belfast Research Portal is retained by the author(s) and / or other copyright owners and it is a condition of accessing these publications that users recognise and abide by the legal requirements associated with these rights.

Take down policy

The Research Portal is Queen's institutional repository that provides access to Queen's research output. Every effort has been made to ensure that content in the Research Portal does not infringe any person's rights, or applicable UK laws. If you discover content in the Research Portal that you believe breaches copyright or violates any law, please contact openaccess@qub.ac.uk. 


\title{
Perspectives of undergraduate module convenors at a South African academic institution on medical student training in evidence-based health care: a qualitative study
}

\author{
Taryn Young, Anke Rohwer, Jimmy Volmink \& Mike Clarke
}

To cite this article: Taryn Young, Anke Rohwer, Jimmy Volmink \& Mike Clarke (2015)

Perspectives of undergraduate module convenors at a South African academic institution on medical student training in evidence-based health care: a qualitative study, South African Family Practice, 57:6, 353-359, DOI: 10.1080/20786190.2015.1090689

To link to this article: https://doi.org/10.1080/20786190.2015.1090689
(c) 2015 The Author(s). Open Access article distributed under the terms of the Creative Commons License [CC BY-NC-ND 4.0]
Submit your article to this journal
曲 Published online: 29 Sep 2015.
ЦIl Article views: 405

View related articles

Citing articles: 1 View citing articles 


\title{
Perspectives of undergraduate module convenors at a South African academic institution on medical student training in evidence-based health care: a qualitative study
}

\author{
Taryn Young ${ }^{\mathrm{ab} *}$, Anke Rohwer ${ }^{\mathrm{a}}$, Jimmy Volmink ${ }^{\mathrm{ab}}$ and Mike Clarke \\ ${ }^{a}$ Centre for Evidence-Based Health Care, Faculty of Medicine and Health Sciences, Stellenbosch University, Cape Town, South Africa \\ ${ }^{b}$ Cochrane South Africa, South African Medical Research Council, Cape Town, South Africa \\ ‘Northern Ireland Network for Trials Methodology Research, Queen's University Belfast, Belfast, Northern Ireland \\ *Corresponding author, email: tyoung@sun.ac.za
}

Background: The increasing disease burden in Africa requires medical graduates to have problem-solving abilities. Interviews were conducted to assess module convenors' perspectives on evidence-based health care (EBHC) teaching and learning.

Methods: Qualitative research methods were used. Faculty convening medical undergraduate teaching modules were purposively sampled. Data collection was done using focus-group or individual interviews. Trained interviewers conducted interviews. Investigators conducted data analysis and interpretation.

Results: Five focus groups and 10 individual interviews were conducted with convenors responsible for theory and clinical modules across various disciplines. Interviewees felt that EBHC teaching and learning were not optimal and indicated varying support for enhancing this. They identified various factors to be considered: contextual factors within the faculty (e.g. recognition for teaching), health sector issues (e.g. clinical workload), access to research, lecturer factors (e.g. competing priorities) and learners (e.g. motivation). They emphasised the key roles of lecturers as role models. Planning together to identify opportunities to integrate teaching and assessment, while ensuring coherence, clear explicit outcomes and promoting faculty development, was regarded as central to strengthening EBHC teaching and learning.

Conclusions: The perspectives of module convenors are key to informing strategies to enhance EBHC teaching and learning.

Keywords: evidence-based health care, medical students, teaching, undergraduate

\section{Introduction}

Medical practitioners need the necessary knowledge, skills and attitudes to function as independent healthcare providers. Furthermore, as part of an interdisciplinary team, they should respond to the unique health needs of the communities they work in. The increasing burden of HIV/AIDS, tuberculosis and chronic diseases, in addition to the major problems of maternal and child health, and injuries, particularly in rural and underserved areas of South Africa, requires increasingly broader problem-solving abilities by new medical graduates. In South Africa, the national accreditation body, the Health Professions Council of South Africa, has adopted a modified CanMEDS framework $^{1}$ as a guide to the key competencies medical doctors should acquire to optimise patient outcomes. It defines the attributes of the graduates according to seven interdependent roles: Medical expert, Scholar, Professional, Communicator, Collaborator, Manager and Health Advocate. This has initiated various curriculum review activities at academic institutions that train medical students.

Embedded in the Scholar attribute is the ability to practise evidence-based health care (EBHC). EBHC has its roots in the field of clinical epidemiology ${ }^{2}$ and was first defined by Gordon Guyatt as 'an ability to assess the validity and importance of evidence before applying it to day-to-day clinical problems.' David Sackett (1996) furthered this definition as 'the conscientious, explicit and judicious use of the current best evidence in making decisions about the care of individual patients', thereby combining clinical expertise and experience with current best evidence obtained from clinically relevant research and patient values. EBHC requires critical thinking, decision-making and an ethos of lifelong learning ${ }^{4}$ and it is recommended that $\mathrm{EBHC}$ should be an integral part of learning in the curriculum of all healthcare professionals. ${ }^{5}$ Learning EBHC is best achieved using clinically integrated teaching and learning with assessment. ${ }^{6}$ Basic concepts successfully introduced in the first year of study ${ }^{7}$ can be reinforced in subsequent years to form a 'golden thread' throughout the medical curriculum. Bedside student learning is particularly useful for learning how EBHC can be applied in practice, but needs point-of-care access to literature, requiring adequate information technology support. The conceptual framework for integrated teaching and learning of EBHC, developed following semi-structured interviews with $24 \mathrm{EBHC}$ programme coordinators from around the world, centres on the engagement between the learner and the teacher within the institutional context. ${ }^{8}$

To inform enhancement of the EBHC curriculum at the Faculty of Medicine and Health Sciences (FMHS), Stellenbosch University, South Africa, we completed a document review of the medical curriculum to assess the learning opportunities and outcomes related to EBHC. ${ }^{9}$ The review captured data on the content, teaching and learning methods, and classified the learning outcomes as knowledge, skills or attitudes. We found that EBHC was covered to varying degrees with teaching confined to specific modules with no evidence of progression of learning. However, this review was based purely on the written information contained in the curriculum module guides, and did not 
necessarily reflect the learning that took place in the classroom, at the bedside and in other settings. For this reason, a subsequent survey of recent medical graduates (students who graduated between 2004 and 2010) explored their perceptions of the relevance and utility of EBHC teaching and learning in the undergraduate medical curriculum. ${ }^{9}$ These recent graduates felt that EBHC was important to cover in the undergraduate curriculum and about a third of respondents felt that medical school training did not prepare them for practising EBHC in the South African healthcare system. They highlighted the need for integration of teaching, especially in the clinical rotations, and suggested that unnecessary duplication of basic concepts should be avoided and attention given to the progression of knowledge and skills gained over the curriculum. ${ }^{9}$

To supplement and enrich the data from the aforementioned studies we carried out an assessment of the perspectives of faculty involved in convening teaching modules on the extent to which the current curriculum includes teaching in EBHC. We aimed to identify best practices and weaknesses in teaching EBHC in the current curriculum, and to identify opportunities for and barriers to strengthening teaching and learning of EBHC.

\section{Methods}

We used qualitative research methods and purposively selected and invited module convenors involved with the coordination of teaching to undergraduate medical students. As the programme runs over six calendar years and includes three phases, we aimed to cover a spectrum of module convenors across various years of study, theoretical and clinical modules, and discipline. The foundation phase covers the first year. The second phase covers the second year to the first semester of the fifth year and includes all theory modules, as well as the early and middle clinical rotations. Student internship is the last phase. We interviewed module convenors from both theory and clinical modules and from a variety of disciplines including family medicine, forensic medicine, haematology, infections and clinical immunology, internal medicine, obstetrics and gynaecology, paediatrics, pharmacology, psychiatry, public health, and surgery. They were all senior academics who were responsible for planning and coordinating teaching and learning within the respective theoretical and/or clinical modules and, with support of other lecturers, also facilitated teaching sessions.

Data were collected in 2013 using five focus-group discussions (FGD). Focus groups were organised according to content areas covered in the curriculum. Three focus groups included five participants each, one focus group included four participants and another included three participants. Where invited participants were not available for FGDs they could opt for an individual interview and if they felt that they were not the appropriate person they could nominate another faculty member within their area of work to participate. We conducted 10 individual interviews. In all instances, participants were informed about the purpose of the interviews and asked to provide written informed consent for participation in the study, for digital recording of the interviews, and for using and disseminating the information gathered.

Trained qualitative researchers conducted the interviews using an interview guide.

\section{Interview Guide:}

- What is your understanding of evidence-based healthcare (EBHC)

-What is your opinion about the relevance or applicability of EBHC to the subject area of your module/s? And how does this relate to the current outcomes of your modules?

- How is the teaching and learning of EBHC covered during your module/s?

-What is your opinion about integrating competencies of EBHC into your modules?

(a) How do you see it being included?

(b) What are the opportunities for including these?

(c) Which teaching methods do you suggest?

(d) Do you envisage any barriers to the incorporation of linked teaching/concepts in EBHC?

(e) Are there facilitators for incorporating it into the module(s)?

Data were captured using a digital voice recorder and additional field notes were taken to ensure full and accurate data capturing. Data were transcribed for analysis purposes. The audiotapes were transcribed verbatim and all transcripts were audited for accuracy by the interviewer who conducted the interview. Names of participants did not appear on the transcriptions. Once the code list was agreed, it guided the subsequent coding process. ${ }^{10}$ Data were imported into Atlas.ti (Atlas.ti $\mathrm{GmbH}$, Berlin, Germany), a software package that facilitates the process of coding qualitative data. The formal coding of all transcripts was completed by one investigator and checked by another investigator. The researchers then carried out the data analysis and interpretation using thematic content analysis to identify key emerging themes, ultimately relating these to each study objective. This iterative process of aggregation and interpretation was undertaken by the lead researcher and discussed with the rest of the research team. During the discussions, these themes and their relevance to the study objectives were highlighted.

This study received ethics approval by the FMHS ethics review committee (N12/11/081). The consolidated criteria for reporting qualitative research (COREQ) ${ }^{11}$ and the standards for reporting qualitative research ${ }^{12}$ guided the reporting of the study.

\section{Findings \\ Current level of EBHC teaching and learning within departments}

In general, participants had good levels of knowledge and understanding of EBHC concepts and its application. Some had misconceptions of EBHC, referring to it for instance as 'citation counting' and that it 'only revolves around the chilly, cold science of evaluation and research' [P1]. Overall, however, participants saw EBHC as a way to enhance and build sound clinical reasoning and critical thinking, and playing an important part in equipping new graduates for the future - to be able to function effectively within any healthcare setting. Opinions varied on the level of current teaching of EBHC, and whether it was adequate or not. Some thought it was unnecessary, and were not really interested in adding EBHC competencies. Others thought that EBHC was being taught but mostly in an implicit, 'intuitive' or 'instinctive' way. This group seemed to feel that students get enough input on 
EBHC in theoretical as well as clinical rotations, even though there are no explicit learning outcomes. Another group acknowledged that the principles of EBHC were being taught, but that these were not carried through effectively to the clinical rotations and to the bedside. They did not think that anything happens intuitively and emphasised the lack of EBHC in clinical practice and ward rounds. They called for a need to link EBHC teaching and learning to appropriate parts of the curriculum and for formalising this input not just in teaching but also in assessments. The role of learning at the bedside in the clinical setting as well as the opportunity to apply the principles was emphasised. They highlighted the value of clinical experience and the importance of assessing applicability of research findings, and also felt that it was not just about facilitating learning about what to do when there was available research but also about what to do in resource constraint settings and in the absence of research evidence. Illustrative quotes are provided in Table 1.

\section{Issues to consider in planning and implementing the teaching and learning of EBHC to undergraduate medical students}

Module convenors raised a number of issues (Table 2) to be considered in planning and implementing EBHC teaching and learning in the undergraduate medical curriculum. These relate to issues within the context/environment of the faculty and the health sector, availability of research evidence, and issues related to the lecturers and the learners. Illustrative quotes are provided in Table 3.

The six-year medical programme is perceived as being full. The view was that each discipline or topic area felt that what they are covering is the most important in the curriculum resulting in a constant 'battle' or competition for space and a resistance to change, irrespective of what the change is. Interviewees felt that teaching staff were overwhelmed with many competing priorities: teaching, research, and heavy clinical workloads. Participants felts that within the context of the faculty's drive for research outputs and the teaching staff's commitment to patient care, teaching often gets marginalised. Change in the curriculum is therefore often seen as additional work, even though it could actually be about working more effectively and efficiently. They mentioned that this resistance to change may also relate to being set in one's ways and not willing to change the way things are done, and to lack of knowledge and skills.

Participants felt that there was lack of continuity within and between modules. This might be as a consequence of having too many lecturers involved where each one comes in and gives a short input on his/her specific topic, often without awareness of what else is covered and thus not linking or connecting to other topics in the same module. The sense was that most people are working in 'silos' and that, to avoid this, there needs to be time for interaction and engagement.

The undergraduate class includes on average 200 students. Within the first six months, this number is doubled as medical and other undergraduate health science students are combined in Phase 1 of the curriculum. Participants highlighted that students' attitudes and perceptions influence learning, and that it is therefore important for students to see the relevance of what they learn as the 'softer' subjects often receive less attention. They mentioned that students want to learn clinical skills and approaches to managing patients and they often do not see the importance of so-called softer skills. From the start, students' perception of what a medical practitioner is, and what the expectations of the programmes are, should be clarified.

Specific to teaching and learning in the clinical setting, participants highlighted that there were too few staff to handle the large student groups. Lecturers found it difficult to give individual attention to students and balance clinical care provision and teaching. They felt that the size of student groups on ward rounds, which typically include undergraduate medical students, student interns, medical specialists in training and

Table 1: Interviewees' perspectives on $\mathrm{EBHC}$ relevance and current level of EBHC teaching

Importance and relevance of EBHC

'To me, this is such an essential skill that our doctors must have, they must have healthy reasoning skills' [FGD2]

'I think that it's very relevant. Perhaps we're not recognising it, for what it is' [P2]

'It's not so much the analysis of the content and the detail but it's actually creating a culture of critical reflection and thinking and asking the right questions.' [FGD4]

'... evidence based healthcare is very, very important no doubt for all the disciplines because you get research in all the different fields and there's good evidence coming out constantly with regards to information that is appropriate to each discipline' [P3]

'... teach them - what is the right way of doing it but I think the better they are equipped in terms of evidence based health, the better, the bigger expert they are, the more they will be able to cope. Rather than to tell them listen we just want you to be average because you are going to work in an average system' [FGD3]

\section{Current teaching and assessment}

... although this is part of the curriculum from the beginning there's no continuity and no scaffolding of these concepts.... The students put it in a box and there's a box in the first year and there's a box in the third year and a box in the fifth year. It's not a golden thread' [FGD1]

'So I think it's evidence based healthcare is actually something that we have been teaching students all along but with the programme that ... is developing we just trying to formalise it a bit more...' [FGD5]

'... the principles of EBHC are not carried through effectively to the clinical rotation years. I feel that this generation of students does not value EBHC so much and the emphasis lies mainly to gain clinical skills to get to the diagnosis. In [clinical module], students focus so much on getting to the diagnosis, they forget about the developing clinical reasoning and processes necessary to get a valid diagnosis.' [FGD2]

'I think it's probably being done, but once again, it's not specifically being said, "These are the competencies we are trying to teach you here". So, in essence, it's almost taught on a subconscious or intuitive manner. The best word to use is an intuitive manner, in which competencies are carried over, in a reading, or a discussion" [P2]

'... we teaching the students tools to think about things which is much more important than the facts ... which gonna change in two or three years' [FGD3] 'In the final exam there is an effort to assess EBHC through critical evaluation of articles around a scenario and assessing the student on how well they incorporate the elements of EBHC into their answers.' [FGD2]

'... evidence based practise is not featuring at the moment...' [P5] 
Table 2: Key issues to consider in planning and implementing EBHC teaching and learning

\begin{tabular}{lll}
\hline Environment/context & Learners & Lecturers / clinicians \\
\hline Faculty & Size of class & Knowledge, attitude and practices \\
Drive for research outputs & Attitude & Teaching approach \\
Lack of recognition for teaching & Learning style & Not enough lecturers \\
'Full' curriculum and competition for space & & \\
Health sector & & Competing priorities \\
Heavy clinical workload & Resistance to change \\
Limited internet access & \\
Limited resources & \\
Research evidence & \\
Availability & Lack of engagement \\
\end{tabular}

consultants, impact on clinical care and on teaching at the bedside.

Participants felt that, within the faculty, lack of recognition of teaching impedes involvement in teaching. The sense was that the faculty was research friendly and that teaching was not recognised at the same level as research. They also felt that the focus of performance assessment was linked to publication output without taking into consideration teaching commitments. There were varying perspectives on leadership support. Some felt that this was adequate while others felt there was a lack of leadership support.

Participants said that environmental constraints to implementing
$E B H C$, e.g. access to the internet, access to electronic databases as well as available resources in the healthcare setting, should be taken into account. They also felt that students should be exposed to various levels of healthcare and pointed out that the realities of the healthcare system in South Africa - busy overloaded public healthcare clinics, huge demand for time and to see many patients - necessitate the availability of experienced teachers in the clinical setting.

EBHC knowledge and attitudes of lecturers were regarded as important considerations in the planning process. Participants highlighted the need for staff development in general and the need to assign suitably qualified lecturers to cover specialised topics, e.g. biostatistics. Both general teaching and learning, as well as EBHC,

Table 3: Issues to consider in planning and implementing EBHC teaching and learning

\section{Environment}

'... trying to integrate to try and get as much of all of it in a short time that they have because their modules are very tight and they have so little time and we are trying to cram so much in a short space of time' [P10]

'I also think that we are faced with the notion that research is rewarded and teaching not. So you would find that sometimes people neglect the softer skills of medicine because there are no rewards in it and spend their attention mainly on research' [FGD2]

'... bedside teaching perspective it is impossible. I mean you've got students standing at the back chatting with each other or being on their cell phones or whatever because nobody can see them they can't hear so they lose interest. You can't shout in a ward you know so that everybody can hear you and so people in the front seven that are standing around the bed and the rest the other seven around them are lost' [FGD3]

'So we have to recognise the constraints and resources and they put pressures on the health system but our training you know for medical students and in fact all in the health professions needs to take into consideration the reality of the ground of the various pressures and it needs to be real, real, real world' [FGD4]

'... practical problems of actual implementation of evidence based healthcare is on a practical level, the assess to resources and you need information technology, computers, access to internet etc. so that is a limitation that we experience on the ground' [FGD4]
\end{abstract}

\title{
Lecturers
}

'... they [clinicians] will see as extra work and a burden. You know and how to convince them that it actually is not extra work nor a burden and that they can actually also benefit from that' [P5]

'... a barrier is the three, the roles that the university expect you to play. It's the researcher, the clinician and the teacher and just the university pushes research so much the clinician, you have your job that you have to do daily so that teaching is always left ...' [FGD4] 'it comes back to living in the real life, there's teaching and there's work where I actually have to be, you know patient care and so on' [P4]

'I regard it as golden threads that should be going through all the departments and all the disciplines but it doesn't always happen. Not because there is no will for it to happen but because there is simply no staff' [FGD4]

'... you know some of you don't seem to be speaking to each other, if we're not speaking to each other as divisions you know, what do the students gain at the end of the day' [FGD4]

'... role models that they see in actual daily life in the hospital is sometimes not up to the standard that you would expect' [P9]

'That knowledge of the lecturers themselves is probably the only barrier because students are very receptive you know if you teach them stuff you know and they do very well in small group teaching' [P7]

\section{Learners}

'... students are making these noises about their lack of interest in what they perceive as softer' [FGD1]

'huge gap between the matric students, the learning methods and what we expect when they come to university and some of the A average students don't know the correct learning methods. They struggle when they come ...' [FGD4] 
Table 4: Advice on how to strengthen teaching and learning of EBHC

\begin{abstract}
Plan together to integrate
'... I mean if it's intuitive, it's not necessarily planned and it needs to be planned. And it cannot be planned in isolation, it must be planned in terms of what has happened before and what is going to happen further on ...' [P6]

'Perhaps there are certain modules, which lends itself more to that. To integrate that, and probably, it's better to integrate that, throughout the entire course, and not just, as a free-standing module or block. We have to look where all of this fits in, and where we can integrate this into existing modules. To get an extra week for something separate, I know, is virtually impossible, or very difficult' [P2]

'... needs to be discussed so that you can review how are you actually teaching it and can you, you know, change your ways to put more emphasis to bring it up in ward rounds' [FGD9]

\section{Ensure coherence}

'A little here and a little there but then eventually there's no coherence, and it's about ensuring that it is visible and it is relevant and it is not seen as there comes the crazy whatever again you know.... So that's it not standard loading little bits but it's actually puzzle pieces of the same picture' [P5]

'I don't want to call it a block it needs to be a house. So you'll need to start with a foundation and have that foundation assessed in the first year then you build the walls and then you put on the roof and so it's not, it's not a once-off big block in the third year or wherever you want to place it. You need to start with foundational skills and have those assessed ... then it should be continuously be built upon in the second year, in the third year, in the fourth and as you go along and then those concepts must be integrated ... infused into the rest of the modules' [FGD1]
\end{abstract}

\title{
Aligning teaching, learning and assessment methods with outcomes
}

'But once you've decided okay what should be covered then you should also decide how it should be covered because certain things are maybe best sorted by lecture, other things students will learn better by experience or by case studies or by doing assignments or whatever, there are so many different ways of engaging students in learning. But you have to identify the best and most appropriate way to do that for a specific topic or specific concept and then also to assess it appropriately. I mean a classic example if some, if you want to know whether somebody can put up a drip. You should ask them to put up a drip, not to write an essay on how to put up a drip you know' [P5]

\section{Assessment drives learning}

'I think at the moment the students are anyway doing an end of task assignment. Where they do have to identify cases or problems that they've picked up while they were rotating in the unit and they already have some questions and we will likely just try and integrate the question' [FGD5]

'You see some of them actually do but we don't see evaluation of it, you see like students are not being assessed in those things, as a component of those modules, and then they were not taken seriously' [FGD1]

\section{Need role models}

'At the end of the day, the role-model speaks much louder than whatever lessons they have learned' [FGD2]

'So it's really good, you know, that you have a prominent, you know, physician who is actually modelling good behaviour to students and teaching that and which is being supported by our faculty' [FGD4]

practices influence the learning process. Current lecturers may not have received training in $\mathrm{EBHC}$, may not be sensitised to $\mathrm{EBHC}$, and most of all may not be practising in an evidence-informed way, resulting in negative role modelling in the clinical setting.

\section{Advice on how to strengthen teaching and learning of EBHC}

Key advice on how to strengthen $\mathrm{EBHC}$ teaching and learning drew attention to the need to plan together, integration of EBHC teaching and learning, ensuring coherence, aligning teaching and learning methods with outcomes, being aware that assessment drives learning and the importance of faculty development (Table 4).

Participants highlighted the need for integration in the curriculum but also with patient care. In working towards integration in the curriculum, it is important to work with convenors to identify opportunities to integrate the learning of $E B H C$. As graduate attributes (including proficiency in EBHC) had been agreed by the medical programme curriculum committee this provided an opportunity for review of the curriculum and created a window to propose changes. Drawing on principles of change management, participants highlighted the importance of involving, and sensitising, consultants and registrars in the various disciplines and convincing them that teaching $\mathrm{EBHC}$ is not necessarily extra work.

Participants emphasised the need for clear explicit learning outcomes and for determining the core content to be covered. Teaching and learning need to build on what has been covered before and need active reinforcement. In order to do this, it is important to engage and work together with module convenors to determine what is covered when, and how one can build on what has been covered, how EBHC can link with current content (thus ensuring relevance), how the theoretical principles can be translated and used in the clinical setting through practical application, and how EBHC can be included in existing assessments within the respective module. In planning assessments, it should not just be about assessing knowledge but also about assessing skills and attitudes. Most importantly, there needs to be alignment between teaching and assessment methods and the learning outcomes.

Successful implementation requires a critical mass of lecturers, from various disciplines, who are familiar with the rest of the module and with what is covered in other modules. Positive role modelling by lecturers and clinicians in the clinical setting is very important. This calls for dedicated faculty development initiatives and also training of future trainers through incorporating EBHC teaching and learning in postgraduate medical specialist training Most importantly, convenors highlighted the need for a common practice, a common language, and a common way of doing things in an evidence-informed way - 'creating a culture of critical reflection and thinking and asking the right questions' [FGD4].

\section{Discussion}

Curriculum change and renewal are ongoing processes, and the implementation of the graduate attributes is a key driver in this current renewal process globally. Curriculum assessment provides information on the current status of teaching and learning, and includes obtaining perspectives from various stakeholders. This study, which focused on the perspectives of module convenors involved with undergraduate medical 
students on EBHC teaching and learning, adds value to the findings of the document review and the survey of recent graduates conducted at FMHS, Stellenbosch University. The three components together provide a clear map of what is covered in the curriculum, when it is covered and what the gaps are. ${ }^{13}$

The document review and survey of recent graduates ${ }^{9}$ found that $E B H C$ teaching was fragmented and recent graduates called for increased teaching of EBHC competencies. In this study, senior academics interviewed also felt that EBHC teaching and learning were not optimal and indicated varying support for the enhancement of EBHC learning in the undergraduate curriculum. In planning and implementing EBHC teaching and learning, they emphasised that key contextual and environmental factors within both the faculty (e.g. faculty support and recognition) and the health sector (e.g. clinical workload, access to internet and computers) must be considered. These link some of the local realities to that of the conceptual framework developed as part of interviews with international programme coordinators. ${ }^{8}$ In addition, the availability of research evidence, and attributes of the lecturers (e.g. EBHC knowledge, skills and practices) and the learners must be considered. The interviewees emphasised the key role of the lecturer, in both theory and clinical modules, as a facilitator and a role model. Planning together, to identify opportunities to integrate teaching and assessment, while ensuring coherence, clear and explicit outcomes and faculty development were central to the advice on strengthening EBHC teaching and learning. In the current era characterised by major advances in the use of information communication technology it was surprising that the views expressed were so silent on the use of technology and how this can be used to facilitate learning, especially once the foundation knowledge has been laid.

The teaching and learning approach of laying the foundation in the early years of the curriculum and building on this through application within clinical modules resonates with best strategies for teaching $\mathrm{EBHC}^{6,14,15}$ In a similar local study among academic healthcare practitioners in South Africa, Mclnerney et al. ${ }^{16}$, using quantitative methods, also identified a need to incorporate EBHC into teaching, perceived 'implicit' teaching of EBHC and found that perceived barriers to the use of EBHC included workload, competing priorities and lack of EBHC knowledge. A study among nurse educators in the United States ${ }^{17}$ also highlighted the need for faculty development to have competent lecturers facilitating the teaching and learning of EBHC. Importantly, various contextual factors - at both faculty and health system level - must be considered in the planning, implementation and evaluation of teaching and learning strategies. ${ }^{8,18}$

Our qualitative study aimed to obtain the perspectives of module convenors and does not reflect the views of all lecturers. Not all invited participants participated in interviews as some indicated that they were'too busy'. It could be that these module convenors have different perspectives from those who agreed to be interviewed. As the lead author (and principal investigator for this study) is very active in promoting EBHC teaching and learning at the faculty where the study was done, we were concerned that her presence in the interviews might overly influence what the participants were willing to say. Therefore, to minimise this potential for bias, the interviews were conducted by trained qualitative researchers. However, because they were not experts in EBHC, potential areas for further probing may have been missed.

\section{Conclusions}

The perspectives of module convenors are key to informing strategies to enhance EBHC teaching and learning - in identifying opportunities for as well as barriers to strengthening teaching and learning of EBHC.

Acknowledgements - The authors would like to thank the Centre for Research on Health and Society, Community Health, Stellenbosch University, for conducting the interviews and arranging the transcriptions. They thank Stellenbosch University Rural Medical Education Partnership Initiative (SURMEPI) working group for the assessment of the medical curriculum on teaching and learning in EBHC, Public Health, Health Systems and Services Research, and Infections Prevention and Control: Ms Anke Rohwer, Prof Taryn Young, Prof Lilian Dudley, Dr Fidele Mukinda, Dr Neil Cameron, Dr Bart Willems, Prof Shaheen Mehtar, Dr Frederick Marais, Dr Angela Dramowski, Prof Ben van Heerden.

Author contributions - Taryn Young (TY) developed the protocol, facilitated the conduct of the interviews, coded the data, conducted the analysis and interpretation, and wrote the manuscript. Anke Rohwer (AR) contributed to protocol development, checked the data coding, interpreted the data and contributed to the manuscript. Jimmy Volmink (JV) and Mike Clarke (MC) approved the protocol, contributed to data interpretation and the manuscript development. All authors approved the final version of the manuscript.

Funding - This research is supported in part by the National Research Foundation of South Africa (UNIQUE grant number 86420). TY and AR are supported in part by the Effective Health Care Research Consortium, which is funded by UKaid from the UK Government Department for International Development, http://www.evidence4health.org, and by the US President's Emergency Plan for AIDS relief (PEPFAR) through HRSA under the terms of T84HA21652 and via the Stellenbosch University Rural Medical Education Partnership Initiative (SURMEPI). MC is Director of the All Ireland Hub for Trials Methodology Research, supported by the UK Medical Research Council (G0901530). The funders had no role in study design, data collection and analysis, decision to publish, or preparation of the manuscript

\section{Conflicts of interest - No known conflicts of interest.}

\section{References}

1. Frank J. The CanMEDS 2005 physician competency framework. Better standards. Better physicians. Better care. Ottawa: The Royal College of Physicians and Surgeons of Canada; 2005.

2. Sackett D. Clinical epidemiology. J Clin Epidemiol. 2002;55:1161-6.

3. Dawes $M$, Summerskill W, Glasziou $P$, et al. Sicily statement on evidence-based practice. BMC Med Educ. 2005;5(1):1-7.

4. Gruppen LD, Rana GK, Arndt TS. A controlled comparison study of the efficacy of training medical students in evidence-based medicine literature searching skills. Acad Med. 2005;80(10):940-4.

5. Glasziou P, Burls A, Gilbert R. Evidence based medicine and the medical curriculum. BMJ. 2008;337:704-5.

6. Young T, Rohwer A, Volmink J, et al. What are the effects of teaching evidence-based health care $(\mathrm{EBHC})$ ? Overview of systematic reviews. PLoS ONE. 2014;9(1):e86706.

7. Srinivasan $M$, Weiner $M$, Breitfeld $P P$, et al. Early introduction of an evidence-based medicine course to preclinical medical students. J Gen Intern Med. 2002;17(1):58-65.

8. Young T, Rohwer A, van Schalkwyk S, et al. Patience, persistence and pragmatism: experiences and lessons learnt from the implementation 
of clinically integrated teaching and learning of evidence-based health care - a qualitative study. PLoS ONE. 2015;10(6):e0131121.

9. Rohwer A, Willems B, Young T. Taking stock of evidence-based health care in the undergraduate medical curriculum at Stellenbosch University: combining a review of curriculum documents and input from recent graduates. Afr J Health Prof Edu. 2015;7(1 Suppl 1):98104. doi: 10.7196/AJHPE.501.

10. Saldaña J. The coding manual for qualitative researchers. London: Sage; 2012.

11. Tong A, Sainsbury P, Craig J. Consolidated criteria for reporting qualitative research (COREQ): a 32-item checklist for interviews and focus groups. Int J Qual Health Care. 2007;19(6):349-57.

12. O'Brien BC, Harris IB, Beckman TJ, et al. Standards for reporting qualitative research: a synthesis of recommendations. Acad Med. 2014; 89(9):1245-51. doi: 0.1097/ACM.0000000000000388.

13. Harden RM. AMEE, Guide No. 21: curriculum mapping: a tool for transparent and authentic teaching and learning. Med Teach. 2001;23(2):123-37.
14. Khan K, Coomarasamy A. A hierarchy of effective teaching and learning to acquire competence in evidenced-based medicine. BMC Med Educ. 2006;6:59. doi: 10.1186/472-6920-6-59.

15. Straus S, Richardson W, Glasziou P, et al. Evidence-based medicine: how to practice and teach EBM. 3rd ed. Edinburgh: Churchill Livingstone; 2005.

16. McInerney P, Suleman F. Exploring knowledge, attitudes, and barriers toward the use of evidence-based practice amongst academic health care practitioners in their teaching in a South African university: a pilot study. Worldviews Evid Based Nurs. 2010;7(2):90-7.

17. Melnyk BM, Fineout-Overholt E, Feinstein NF, et al. Nurse practitioner educators' perceived knowledge, beliefs, and teaching strategies regarding evidence-based practice: implications for accelerating the integration of evidence-based practice into graduate programs. J Prof Nurs. 2008;24(1):7-13.

18. Mi M. Factors that influence effective evidence-based medicine instruction. Med Ref Serv Q. 2013;32(4):424-33. 